\title{
Differential Thermal Analysis of Commercial and Dental Waxes
}

R. G. CRAIG, J. M. POWERS, and F. A. PEYTON

School of Dentistry, University of Michigan, Ann Arbor, Michigan

Several authors ${ }^{1,2}$ have reported differential plots of the rate of cooling versus the temperature of waxes. Lange and Jochinke, ${ }^{3}$ Smith et al., ${ }^{4}$ Lorant, ${ }^{5}$ and Uchida ${ }^{6}$ used differential thermal analysis for examining phase changes in a variety of waxes, whereas Martin et al. ${ }^{7}$ studied infrared absorption as a method for determining phase changes.

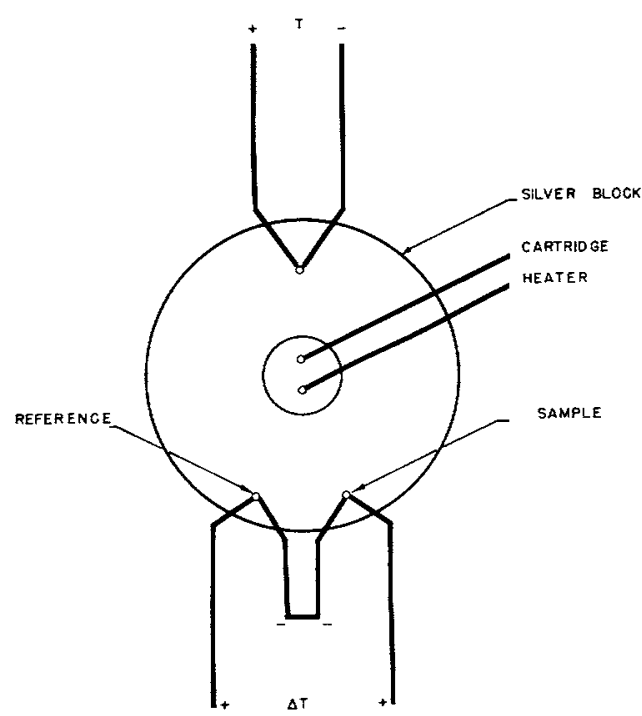

FIG. 1.-Schematic sketch of the differential thermal analysis (DTA) cell.

Since phase changes generally result in dimensional changes, the temperatures at which the phase changes occur in waxes and the influence of adding one wax to another are important in understanding the behavior of waxes. Differential thermal analysis (DTA) offers a useful tool in the study of phase transitions. This study examines the temperature of phase transitions for various commercial, dental, and combined waxes.

This investigation was supported by USPHS Research Grant DE-01234 from the National Institute of Dental Research, National Institutes of Health, Bethesda, Md. Presented at the 45th General Meeting of the IADR, Washington, D.C., March 18, 1967.

Received for publication March 28, 1967.

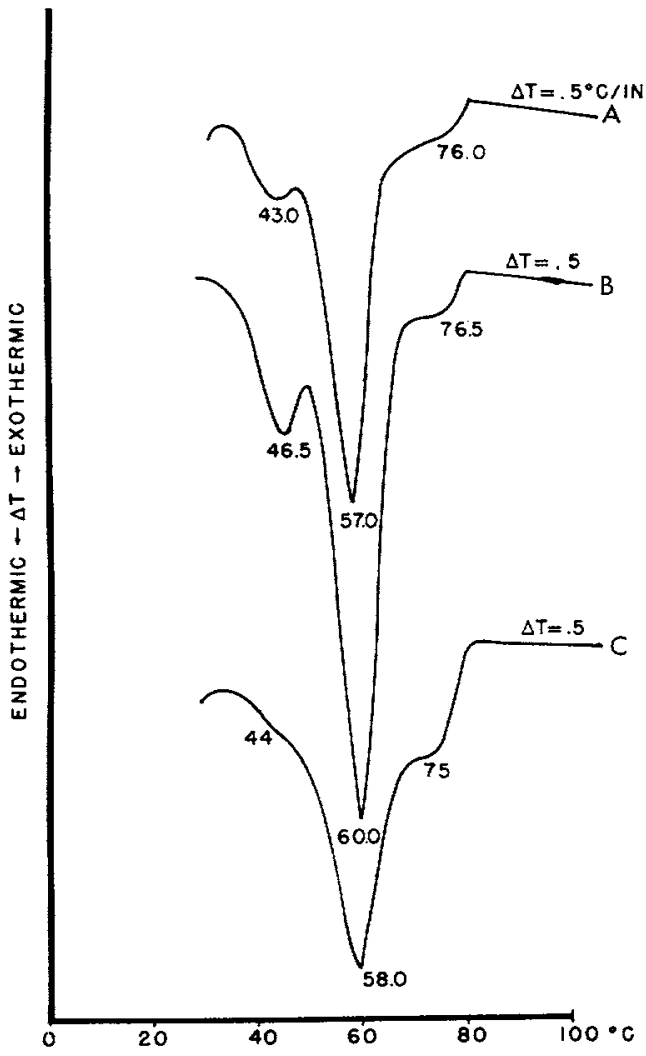

FIG. 2.-The DTA curves of paraffin wax at three rates of heating. (Note: One inch on the vertical scale of all thermograms is equivalent to the distance from 0 degrees to 20 degrees on the horizonta? axis.)

\section{Materials and Methods}

The waxes were subjected to differential thermal analysis as obtained from the supplier* or blended as indicated

Differential thermal analysis $\dagger$ was carried out from $25^{\circ}$ to $200^{\circ} \mathrm{C}$. for the various

* Ross Company, Jersey City, N.J.

t DuPont 900 Differential Thermal Analyzer, E. I. duPont de Nemours \& Co. (Inc.), Instrument Products Division, Wilmington, Del. 19898. 


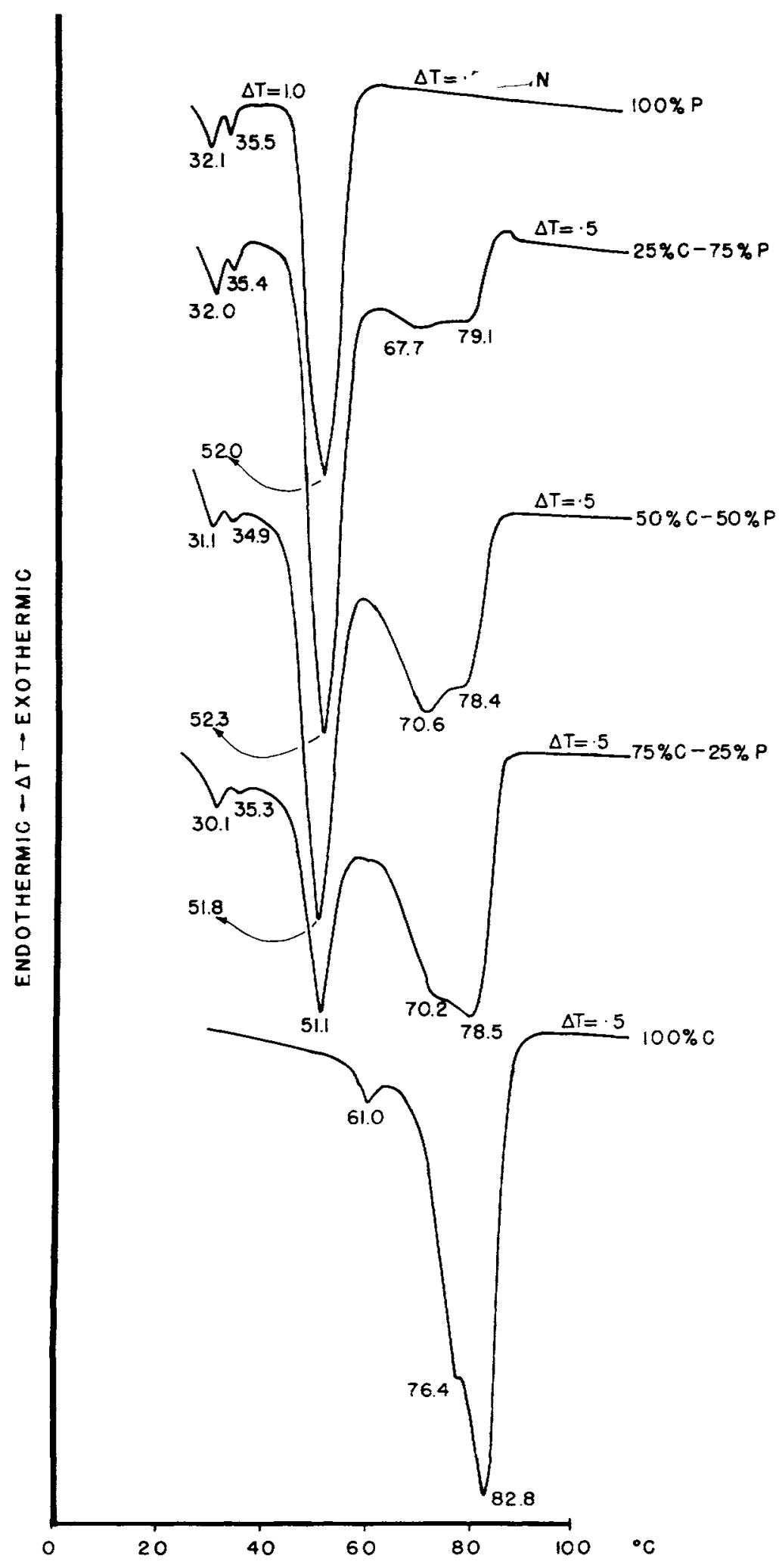

FIG. 3.-The DTA curves for paraffin and carnauba wax and combinations containing these waxes. 
waxes. The analysis was conducted in a nitrogen atmosphere, and a heating rate of $20^{\circ} \mathrm{C}$. per minute was used except in two selected runs in which rates of $5^{\circ}$ and $10^{\circ} \mathrm{C}$. per minute were chosen.

The thermal analysis cell is shown in Figure 1 . The reference thermocouple was placed in glass beads and was connected to the sample thermocouple so that the difference in temperature $(\Delta \mathrm{T})$ was recorded. A third thermocouple was placed at a symmetrical position in the cell to record the temperature $(\mathrm{T})$. The output from these thermocouples was fed into an $\mathrm{x}-\mathrm{y}$ recorder which gave plots of $\Delta \mathrm{T}$ versus $\mathrm{T}$. When the reference and the sample were heated at the same rate, a plot of $T$ versus $\Delta T$ gave a straight horizontal line, but when the sample absorbed heat more slowly than did the reference as a result of a transition, a decrease in $\Delta \mathrm{T}$ occurred. This decrease resulted in a peak with a minimum at the transition temperature and indicated an endothermic change. If the transition is exothermic, a maximum peak is observed.

\section{Results}

Thermograms of paraffin wax* are shown in Figure 2 for rates of heating of $5^{\circ}, 10^{\circ}$, and $20^{\circ} \mathrm{C}$. per minute. Higher rates of heating increased the sensitivity of the thermograms but decreased the resolution of the solid-solid transformations at $32^{\circ}$ and $35^{\circ} \mathrm{C}$. The solid-liquid transformation or melting point of paraffin occurred at $52^{\circ} \mathrm{C}$. The heat of solid-solid transitions was $11.3 \mathrm{cal} . / \mathrm{Gm}$., and the heat of melting was $42.3 \mathrm{cal} . / \mathrm{Gm}$. for paraffin wax.

Thermograms of paraffin and carnauba wax and combinations of these waxes are shown in Figure 3 ; those of paraffin and beeswax are given in Figure 4 . The peak temperatures represent the means of five replications of the thermograms. Analyses of variance were calculated for paraffin and combinations containing carnauba wax as well as for carnauba wax and combinations containing paraffin. The analysis for paraffin and combinations with paraffin showed that the values of the peak temperatures characteristic of paraffin varied for the different waxes at the 0.01 level of confidence $\left(\mathrm{F}_{3,48}=31.1>4.31\right)$. Ranking was done according to Duncan's new multiple range

\footnotetext{
* Ross fully refined paraffin wax, manufactured by
} Ross Company, Jersey City, N.J. test $^{8}$ and, when all three peaks were used for 100 percent paraffin and a combination of 75 percent paraffin and 25 percent carnauba wax, they were statistically different than those for the combinations containing 50 and 75 percent carnauba wax at the 0.01 level. When only the melting transition was considered, the mixture containing 75 percent carnauba wax was the only sample that differed (about $1.5^{\circ} \mathrm{C}$. lower) from the combinations containing carnauba wax or from paraffin at the 0.01 level.

The analysis of variance for the peak temperature as a function of waxes was determined for carnauba wax and combinations containing paraffin wax. An $\mathrm{F}_{3,32}$ of $\mathbf{1 1 5 . 5}$ was greater than 4.51 , the critical value at the 0.01 level, which showed that the peak temperatures were different for these waxes. Ranking, as before, showed that combinations of carnauba and paraffin wax had the same peak temperatures but that the peak temperatures for pure carnauba wax were different (approximately $5^{\circ}$ to $6^{\circ} \mathrm{C}$. higher)

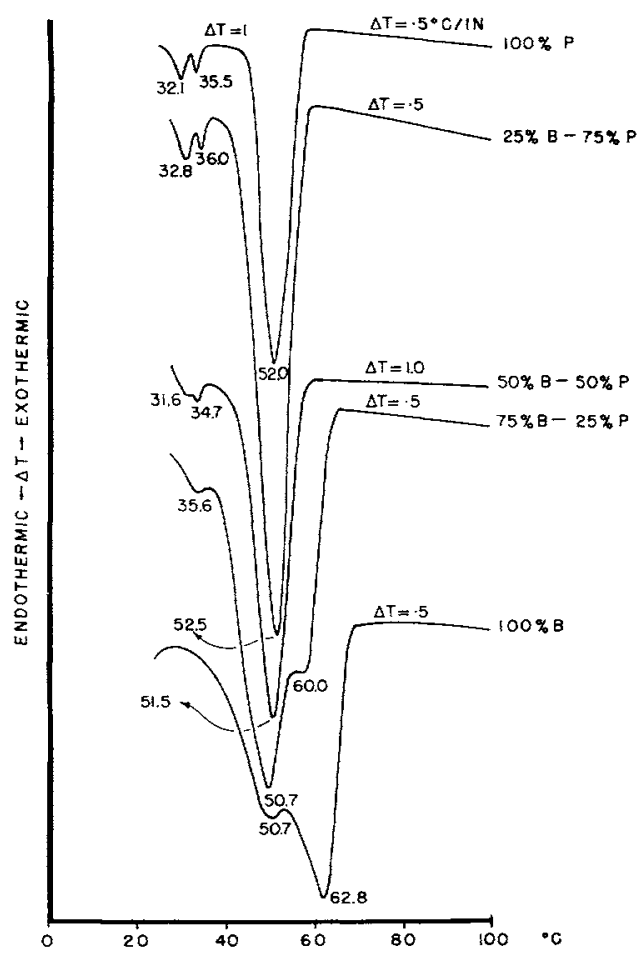

FIG. 4.-The DTA curves for paraffin and beeswax and combinations containing these waxes. 
from those of the combinations at the 0.01 level.

An analysis of variance for the peak temperatures associated with paraffin was determined for pure paraffin and for combinations containing beeswax and those associated with beeswax containing paraffin. $\mathrm{F}_{2,42}$ of
0.37 and $F_{1,18}$ of 0.30 compared with critical values of 5.18 and 8.29 , respectively, at the 0.01 level establish that additions of beeswax to paraffin did not significantly influence the transition points characteristic of paraffin and vice versa. In spite of these results, the thermogram for 75 percent beeswax and 25

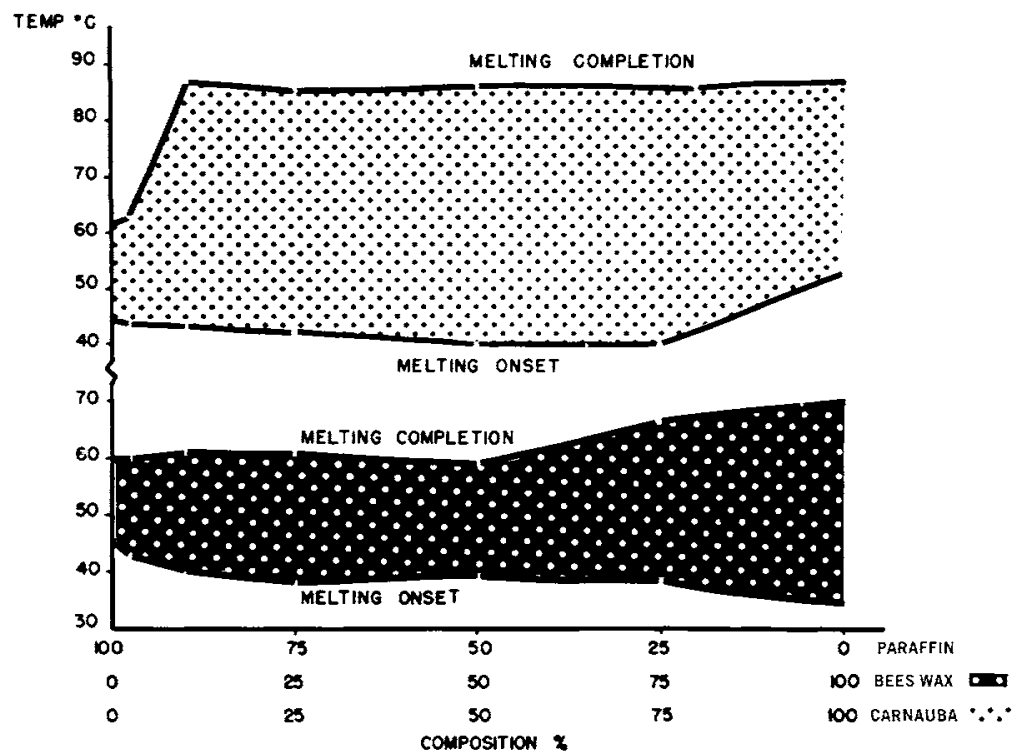

FIG. 5.-Onset and completion of melting temperatures for paraffin wax mixed with carnauba wax or beeswax.

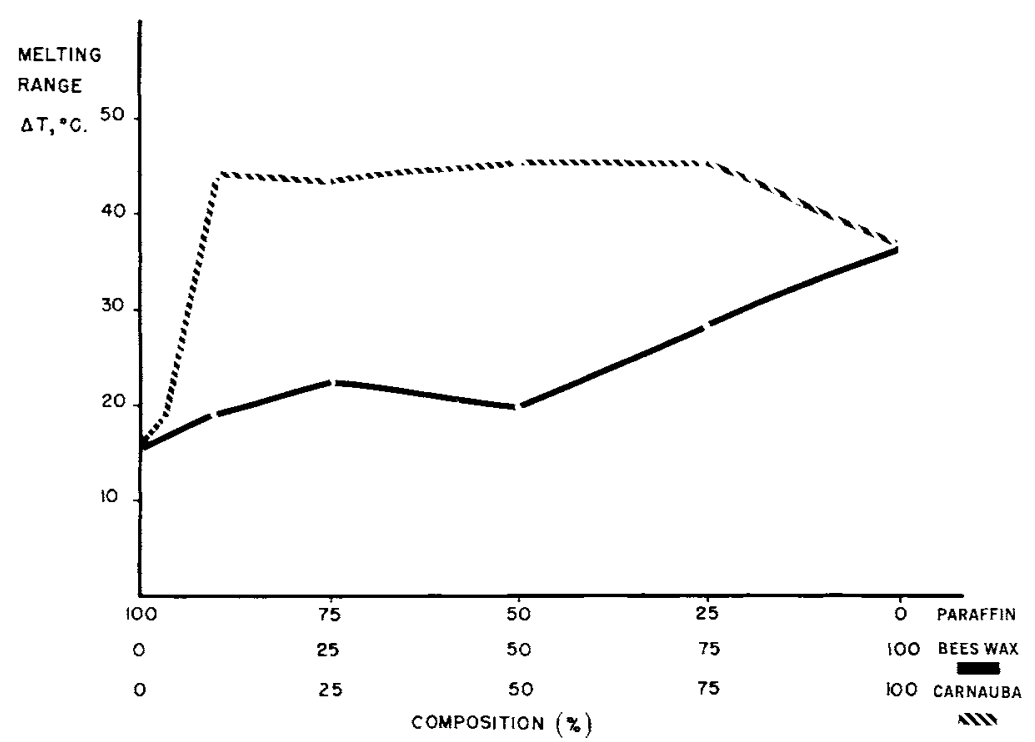

FIG. 6.--Melting range of paraffin wax combinations containing carnauba wax or beeswax as determined from DTA curves. 
percent paraffin had peaks characteristic of both waxes whereas, in the thermogram for 50 percent each of beeswax and paraffin wax, the paraffin wax suppressed the peaks associated with beeswax.

The effect of additions of carnauba or beeswax to paraffin wax on the onset and completion of melting, as indicated by the thermograms, is shown in Figure 5, and the melting ranges for the various combinations are given in Figure 6 . The most noticeable features of these data are the great effect of small additions of carnauba wax on increasing the melting range. Also, major additions of carnauba or beeswax to paraffin wax had little influence on the onset of melting, and beeswax had less effect on the completion of melting than did carnauba.

Thermograms of ester waxes, candellila, ouricury, and montan, are shown in Figure 7 , with melting transitions at $65^{\circ}, 82^{\circ}$, and $83^{\circ} \mathrm{C}$., respectively. Candellila and ouricury wax had a melting range of $33^{\circ} \mathrm{C}$., whereas montan wax had a melting range of $45^{\circ} \mathrm{C}$.

The thermograms of four hydrocarbon waxes-barnsdahl, ceresin, litene, and polyethylene - are given in Figure 8. Barnsdahl wax is characterized by a single transition at $86^{\circ} \mathrm{C}$. and a broad melting range of about $58^{\circ} \mathrm{C}$. Litene has two transitions, at $54^{\circ}$ and $72^{\circ} \mathrm{C}$., and a narrower melting range of $30^{\circ} \mathrm{C}$. Ceresin and polyethylene waxes had numerous transitions and broad melting

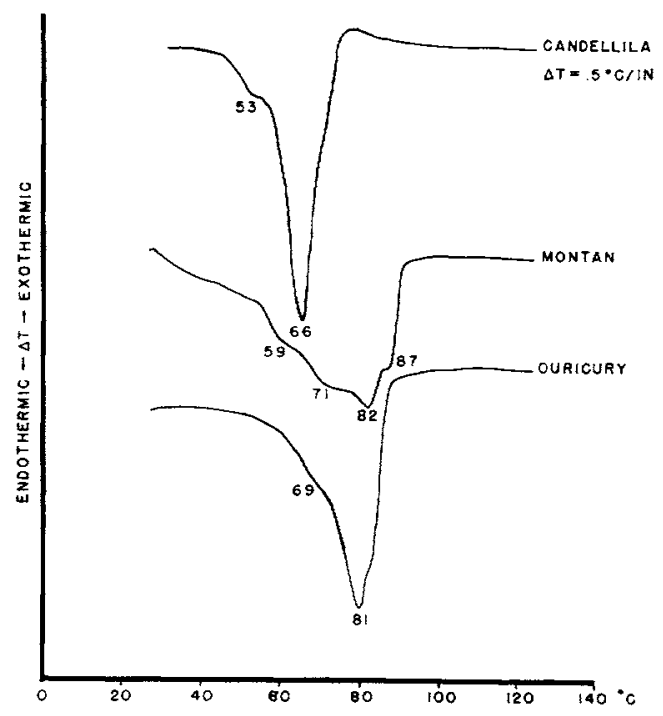

Fic. 7.-The DTA curves for ester waxes. ranges of $45^{\circ}$ and $60^{\circ} \mathrm{C}$., respectively. Ceresin wax had transitions at $34^{\circ}$ and $37^{\circ} \mathrm{C}$. which were reminiscent of those of parafin wax.

The thermograms of three dental inlay waxes $(A, * B,+C+)$ are shown in Figure 9 . The hydrocarbon waxes used in their compounding had melting transitions between $58^{\circ}$ and $60^{\circ} \mathrm{C}$.

\section{Discussion}

Comparison of published thermal analysis measurements ${ }^{1-6}$ for paraffin wax indicates a similarity with those attained in this study except, previously, none or one rather than two solid-solid transitions were reported. The two solid-solid transitions may be explained on the basis of the crystal structure of paraffin wax. ${ }^{9-11}$ As shown by radiographic studies, paraffin wax crystallizes from the melt in hexagonal close-packed structure with the long axis perpendicular to a basal plane. Odd-numbered paraffin hydrocarbons transform from hexagonal to orthorhombic with the long axis perpendicular to the basal plane. Low and high molecular weight even-numbered paraffin hydrocarbons transform to triclinic or monoclinic lattice. Thus, the two solid-solid transitions could result in a parafin wax having a combination of odd-numbered and even-numbered hydrocarbons that transform to the hexagonal lattice on heating, thus giving only one solid-liquid transition.

More transitions were detected in the present study than in previously reported studies of hydrocarbon and ester waxes, thus indicating the improved sensitivity of the differential thermal analyzer. Interpretation of the various peaks from thermal analysis alone is not possible.

The measurements of melting points, as determined by DTA, indicated that addition of large amounts of carnauba wax to paraffin wax had little effect on the melting point; 50 percent to 75 percent of this wax was added before a detectable change in melting point of paraffin wax was noticed. These findings are in agreement with observations of Craig et al. ${ }^{12}$ but they are not in agreement with those given in current textbooks on waxes

\footnotetext{
* Kerr blue inlay casting wax regular, manufactured by Kerr Mfg. Co., Detroit, Mich.

$\uparrow$ Kerr blue inlay casting wax hard, manufactured by Kerr Mfg. Co., Detroit, Mich.

$\ddagger$ Dr. Feck's purple hard wax, manufactured by Kerr Mfg. Co., Detroit, Mich.
} 


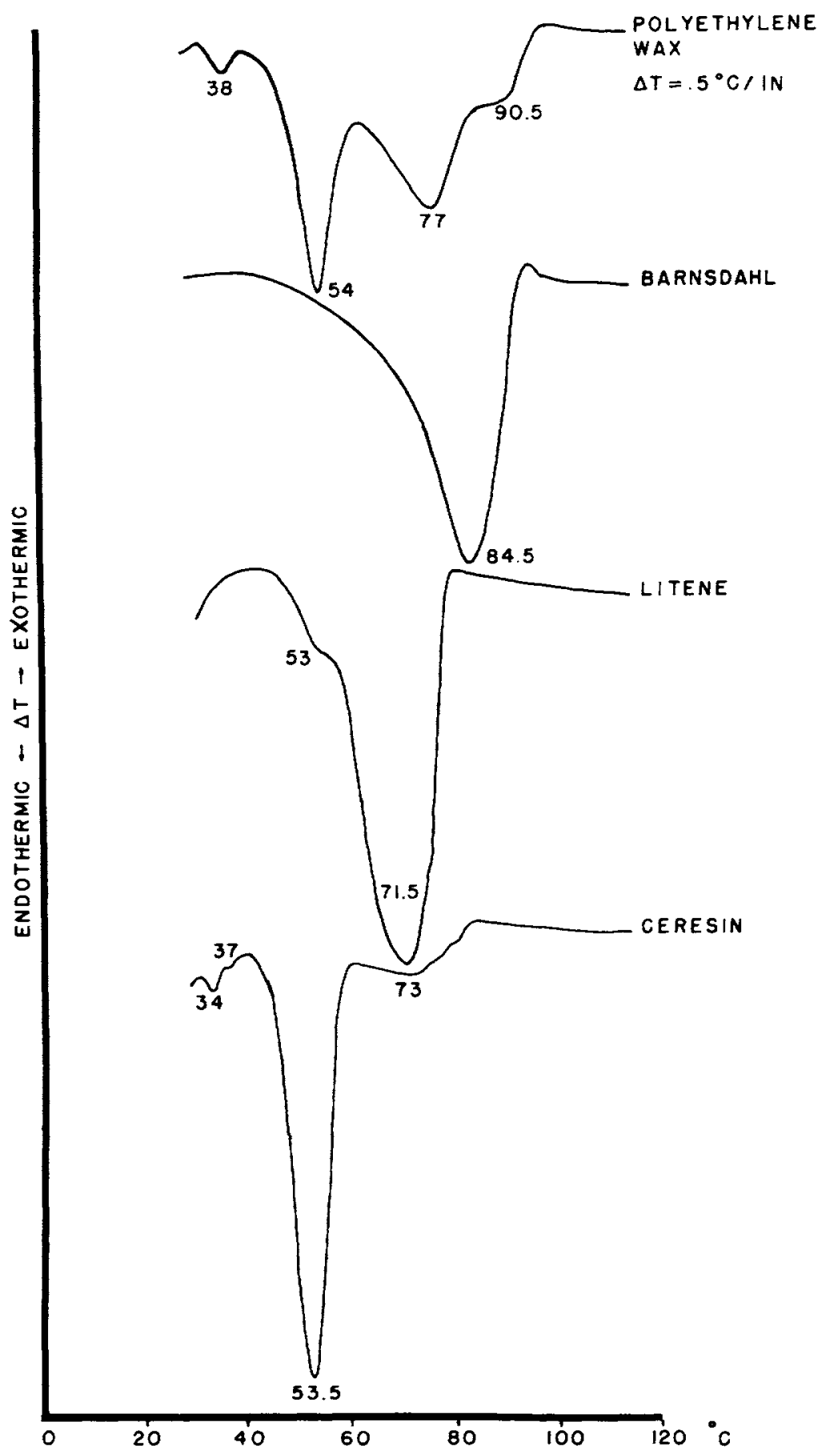

FIG. 8. - The DTA curves for hydrocarbon waxes. 


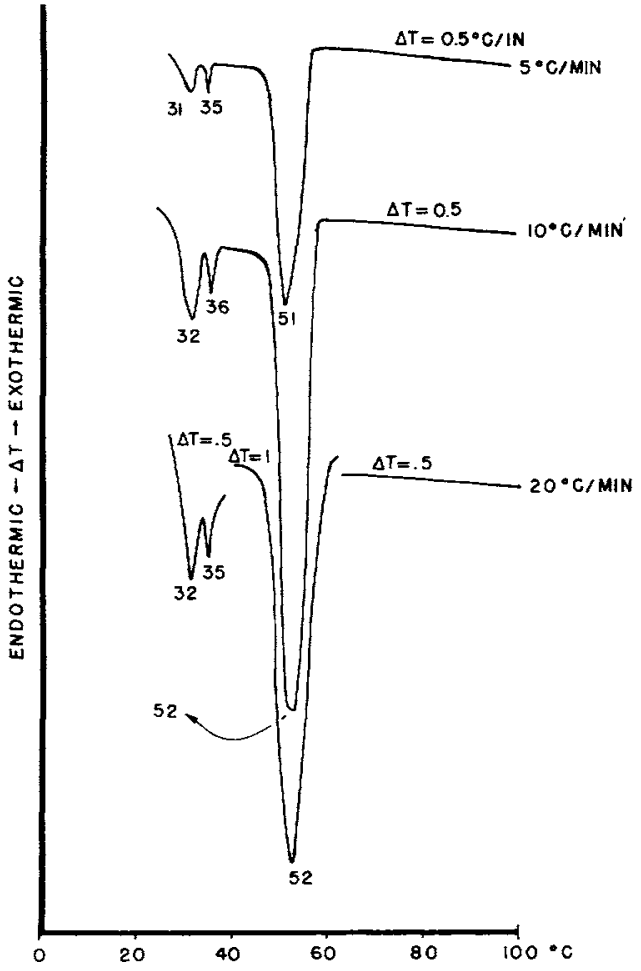

FIG. 9.-The DTA curves for three dental inlay waxes.

and dental materials where it is reported that 2 percent to 3 percent of carnauba wax will increase the melting point of paraffin wax $15^{\circ}$ to $20^{\circ} \mathrm{C}$. The principal result of adding these waxes, particularly carnauba, to paraffin is an increase in the melting range. This combination provides a system in which the paraffin wax melts, but the matrix of carnauba wax provides a viscous solid that can be described as a gel.

The pronounced effects of carnauba wax on the melting point of paraffin reported by Ellis ${ }^{13,14}$ and Koch et al. ${ }^{15}$ appear to be associated with the experimental method in which flow properties and the effect of capillarity between the wax samples and the supporting plate are important considerations. The agreement between previously reported melting points determined by use of the capillary tube ${ }^{1}$ method and the solid-liquid transitions from DTA measurement was excellent. Reported temperature breaks in the thermal expansion curves ${ }^{1}$ for hydrocarbon waxes are in agreement with the solid-solid transition temperatures observed in this study, although the DTA values were $4^{\circ}$ to $6^{\circ} \mathrm{C}$. higher. Ester waxes had solid-to-liquid transformation temperatures $10^{\circ}$ to $20^{\circ} \mathrm{C}$. higher than transitions indicated by the thermal expansion data.

The thermograms for combinations of paraffin and carnauba wax indicated the data could be used to analyze combinations for these ingredients, but similar data for combinations containing parafin and beeswax illustrated that quantitative analyses could not be obtained and qualitative analyses would be in question. As shown in Figure 9, the dental waxes were composed of a hydrocarbon wax with a melting point of $57^{\circ}$ to $60^{\circ} \mathrm{C}$., and of a plant wax (probably carnauba) with a melting point, corrected for the influence of hydrocarbon wax, of $80^{\circ}$ to $82^{\circ} \mathrm{C}$. The repression of the peak at $44^{\circ} \mathrm{C}$. with wax $\mathrm{C}$ suggests the presence of beeswax.

Candellila, ouricury, carnauba, and montan waxes and beeswax had transitions between $50^{\circ}$ and $70^{\circ} \mathrm{C}$. melting the melting transition, which suggests the presence of hydrocarbon waxes. Montan wax had a broad melting range from $25^{\circ}$ to $92^{\circ} \mathrm{C}$. with numerous transitions, suggesting a complex combination of hydrocarbons, alcohols, acids, and esters. Ceresin, litene, barnsdahl, and polyethylene waxes had broad melting ranges, indicating that they were composed of various hydrocarbons. The increased toughness of the microcrystalline waxesbarnsdahl and litene - and polyethylene wax is partially related to the melting range. These waxes could be considered substitutes for paraffin wax when this quality is desired.

\section{Summary}

Transition temperatures from $20^{\circ}$ to $200^{\circ} \mathrm{C}$. have been determined for a variety of waxes and combinations of waxes by differential thermal analysis (DTA). Additions of carnauba wax to paraffin wax influenced the transitions of the latter when the former was present in large concentrations; 75 percent carnauba in paraffin wax lowered the melting transition about $1.5^{\circ} \mathrm{C}$. Additions of 25 percent paraffin to carnauba wax decreased the melting transition of the latter about $5^{\circ}$ to $6^{\circ} \mathrm{C}$.; further increase in paraffin wax above 25 percent did not alter the melting transition associated with carnauba wax. No influence on the transitions was observed with combinations of paraffin and beeswax, 
although 50 percent or more of paraffin wax completely suppressed the transition associated with beeswax.

The major influence of small additions of carnauba to paraffin wax was a pronounced increase in the melting range; addition of beeswax to paraffin wax did not produce comparable results.

The three transition temperatures of paraffin wax have been interpreted in terms of crystal structure from published radiographic values. The two solid-solid transitions at $32^{\circ}$ and $35^{\circ} \mathrm{C}$. have been interpreted to represent the transformation of some hydrocarbons from hexagonal close-packed to orthorhombic structure and others from hexagonal to monoclinic or triclinic structure.

Thermograms for dental waxes were interpreted in terms of those obtained for natural waxes. It was observed that DTA would not be suitable for quantitative analysis and, at times, questionable for qualitative analysis of waxes. Thermograms of natural and one synthetic wax were discussed in terms of their possible use in dental applications.

Melting transitions from DTA were in good agreement with capillary tube melting points, and the solid-solid transition showed a general relationship to discontinuities in thermal expansion curves.

\section{References}

1. Craig, R. G., Etck, J. D., and Peyton, F. A.
Properties of Natural Waxes tised in Dentistry, J. Dent. Res., 44:1308-1316, 1965.

2. Ohashi, Masayoshi, and Paffenbarger, G. C. Melting, Flow, and Thermal Expansion Characteristics of Some Dental and Commercial Waxes, $J A D A, 72: 1141-1150,1966$.

3. LANGE, J., and Jochinke, H. The Characterization of Waxes by Differential Thermal Analysis, Fette, Seifen, Anstrichmittel, 67:89-94, 1965.

4. Smith, D. C., Earnshaw, R., and MCCrorie, J. W. Some Properties of Modelling and Baseplate Waxes, Brit. Dent. J., 118:437-442, 1965.

5. LoRANT, BeLA. Derivatographic Investigation of Fats and Derivatives of Fatty Acids, Seifen-OeleFette-Washse, 92:57-59; 149-150, 1966.

6. Uchida, Taxashi. The Phase Changes of Commercial Paraffin Waxes by Differential Thermal Analysis, Kogyo Kagaku Zasshi, 69:1315-1319, 1966.

7. Martin, J. M. Jr., Johnston, R. W. B., Cannon. H. J., and O'YEAL, M. J. Infrared-Absorption Method for Measuring Phase Transitions of Waxes, Anal. Chem., 30:1005-1006, 1958.

8. Duncan, D. B. Multiple Range and Multiple F Test, Biometrics, 11:1-42, 1965 .

9. EDwards, R. T. Crystal Habit of Paraffin Wax, Indust. Engineer. Chem, 49:750-757, 1957.

10. - . Molecular Arrangement and the Properties of Petroleum Waxes, Tappi, Spec. Tech. Assn. Publ. No. 2, 95-110; 110-111, 1963 .

11. Hatt, H. H., and Lamberton, J. A. Molecular Structure of Waxes, Res. Sci. Appl. Indust. 9:138$146,1956$.

12. Craig, R. G., Eick, J. D., and Peyton, F. A. Flow of Binary and Tertiary Mixtures of Waxes, J. Dent. Res., 45:397-403, 1966.

13. Ellis, W. J. The Melting-Point Curves of Some Binary Wax Mixtures, $J$. Council Sci. Indust. Res. (Australia), 16:179-184, 1943.

14. - Melting Points. II. Melting Points of Binary Wax Mixtures, Petroleum (London), $8: 115$, 1945.

15. Koch, J, R., Hable, G. J, and WRANGeil, LEwis. Melting-Point Studies of Binary and Tertiary Mixtures of Commercial Waxes, Anal. Chem, 10:167168,1938 . 